\title{
Ley de derechos y deberes de las personas en la atención de salud. Una mirada bioética
}

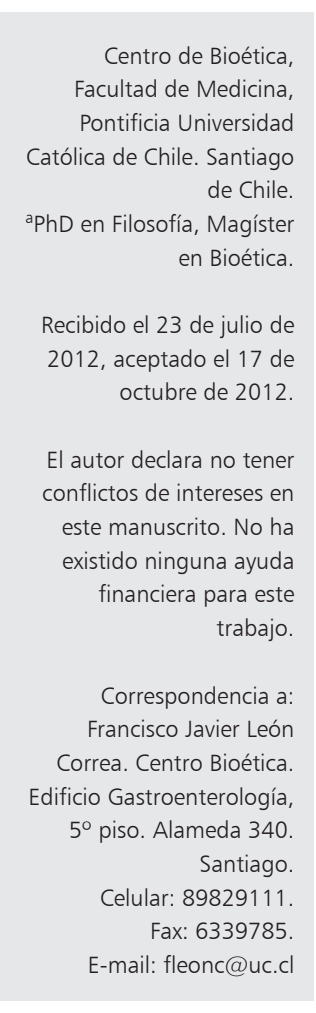

FRANCISCO JAVIER LEÓN C. ${ }^{\mathrm{a}}$

\section{The law of rights and duties of persons in health care from the viewpoint of bioethics}

The enactment of Law 20.584 in April of 2012 promotes a change in the physicianpatient relationship, with recognition of people's rights and duties in healthcare by all the health professional and entities. The legal obligation, and not only the ethical one, as it currently happens, for humane treatment and regard for the dignity of the sick, informed consent, medical data confidentiality, the possibility to reject treatments, etc., is established. This review analyzes the contents of this law, especially those parts affecting physicians. It exposes its limitations, especially those related to minors' consent, living wills and research in people with mental disabilities. It also highlights positive aspects such as the promotion of a humane healthcare. This Law begins to consider patients' autonomy shyly. However, from a bioethical viewpoint, there are still many issues to be perfected such as healthcare humanization, excellence of patient care, healthcare quality and medical professionalism, considering competence, social service, charity and solidarity. It is a first step that must be supplemented with a greater development of medical deontology, and the development of clinical and institutional bioethics.

(Rev Med Chile 2012; 140: 1490-1494).

Key words: Bioethics; Delivery of health care; Ethics committees; Informed consent; Patient's rights.
$\mathrm{E}$ n abril de 2012 se promulgó la Ley 20.584, que regula "los derechos y deberes que tienen las personas en relación con acciones vinculadas a su atención en salud", después de una tramitación parlamentaria de once años. No queremos realizar un análisis jurídico del texto, sino exponer algunos problemas desde la mirada de una ética aplicada interdisciplinar, como es la bioética.

El contexto de la Ley es la reforma de la salud en Chile. En 2001 se planteó un cambio de orientación: desde una visión más ligada al ideario liberal, que favorece la intervención de privados y concibe la misión del Estado en este ámbito como subsidiaria ${ }^{1}$, a una visión que favorece la medicina pública y concibe la salud como un derecho humano $^{2}$ y responsabilidad indelegable del Estado ${ }^{3: 13}$. En el preámbulo de la Ley, escrito por la Presidente de la República, Dra. Michelle Bachelet ${ }^{4}$, se habla de que debe atender a valores y principios éticos de una reforma de salud basada en los derechos de los pacientes. "El principal soporte y aliado (de la reforma) será la incorporación de los derechos explícitos y el empoderamiento ciudadano para que las personas sean sujetos activos en el proceso de salud-enfermedad"5.

El derecho a la salud debe ser atendido "de modo oportuno y sin discriminación", como señala la actual Ley, y hay que adecuar la oferta pública a las necesidades y derechos de las personas ${ }^{5: 268}$. La Ley no establece cuál es la atención oportuna y en qué consistirían las discriminaciones, pero se remite en esto a las demás leyes de la reforma.

En definitiva, viene a sancionar algunos de los 
Ley derechos y deberes de las personas en atención de salud - F. J. León C.

temas debatidos y desarrollados antes por la deliberación en bioética, e incorporados sólo en parte a la atención clínica en Chile. Los derechos de los pacientes están en el debate de "cómo debía ser" -más allá de "cómo era"- la relación del médico con sus pacientes ${ }^{6}$, y de las instituciones de salud con los usuarios de las mismas. La defensa de la autonomía como principio ético, especialmente en la bioética anglosajona, supuso un brusco cambio en una medicina paternalista, fundamentada en la no maleficencia y la beneficencia, y el desarrollo de los sistemas públicos de salud y del derecho a la atención de los ciudadanos cuando enferman, supuso la introducción del principio ético de justicia en la distribución de recursos, de equidad en el acceso y de protección de los más vulnerables o vulnerados en salud. Y siguen siendo deberes éticos fundamentales la no maleficencia y también la beneficencia: proporcionar la mejor calidad asistencial posible, con excelencia profesional e ideales de servicio, solidaridad y compasión, más allá de un profesionalismo distante. Estos aspectos se incorporan ahora al texto legal, como obligaciones jurídicas de los prestadores institucionales e individuales, y no sólo como criterios éticos.

\section{La recepción de la Ley y el contexto latinoamericano}

El texto final ha recibido críticas de dos tipos: los que piensan que no habría que reglamentar los derechos de los pacientes, pues supondrá una mayor judicialización de la medicina, al reconocer el consentimiento informado y la posibilidad de rechazar tratamientos, por ejemplo, y los que consideran que se ha recortado excesivamente el planteamiento inicial en cuanto a la autonomía, como en el caso de las voluntades anticipadas, y que se ha cambiado y se ha dejado cerrada la puerta al enfoque de fondo del reconocimiento de los derechos de los pacientes dentro de una auténtica reforma de salud ${ }^{7}$.

Si la comparamos con otras leyes recientes de países latinoamericanos, es cierto que no reconoce la autonomía del paciente como lo hacen la Ley española (2002), la argentina (2009), o la Carta dos direitos dos usuarios da saude, de Brasil (2006). Ecuador ha aprobado una "Política de la Relación Sanitaria” (2011), con estrategias para la imple- mentación de los derechos y principios bioéticos, más amplia que una mera Ley de derechos, y Perú también acaba de aprobar unos lineamientos para el ejercicio de la bioética desde el reconocimiento de los derechos humanos, aunque aún esté por concretarse.

Aún es pronto para conocer cuáles serán las consecuencias de la aplicación de esta Ley en Chile. Dependerán en gran parte de su difusión y acogida por los profesionales y por los propios pacientes, pero cabe una visión intermedia. Es un paso adelante en un proceso largo que no ha terminado. Se ha aceptado el consentimiento informado -aún con muchas reticencias- pero estamos en el inicio de la participación de los pacientes en las decisiones sobre su atención de salud y sobre el sistema y las políticas de salud, con una primera reglamentación de la participación y con su acceso a los Comités de Ética.

\section{Un modelo humano de asistencia en salud}

La bioética intenta ayudar al establecimiento de una relación interpersonal entre profesionales de la salud y pacientes, de forma que se vea la persona del enfermo de modo integral, no como un "caso clínico" o una sucesión de estudios sistémicos de diversos especialistas, sino desde la visión interdisciplinar de todos los profesionales involucrados en su atención, a través del "médico tratante", al que el paciente debe conocer, pues tiene derecho "a saber quien autoriza y efectúa sus diagnósticos y tratamientos" (Art. 90).

Es cierto que el "derecho a un trato digno" es más una obligación de los profesionales que un derecho específico de los pacientes, pero no nos parece innecesario que socialmente establezcamos claramente que queremos una mejor y más compasiva atención por parte de todos los profesionales de la salud. Las instituciones tienen que velar por que "se utilice un lenguaje adecuado e inteligible durante la atención" (Art. $5^{\circ}$ a), "se adopten actitudes que se ajusten a las normas de cortesía y amabilidad generalmente aceptadas" (Art. $5^{\circ} \mathrm{b}$ ), lo que incluye llamar a los pacientes por su nombre, "respetar y proteger la vida privada y la honra de la persona" y no sacar fotos o filmar sin su previo consentimiento, que debe ser por escrito en caso de publicidad o difusión en los medios de comunicación (Art. $5^{\circ} \mathrm{c}$ ). 


\section{El reto ético del consentimiento informado}

Una parte fundamental está dedicada a la información al paciente, tanto al iniciar una atención en salud o una hospitalización (Art. $8^{\circ}$ ), como durante la misma (Art. $10^{\circ}$ ) o en el momento de dar el alta médica (Art. 11\%). Información "oportuna y comprensible, por parte del médico u otro profesional tratante... acerca del estado de su salud, del posible diagnóstico de su enfermedad, de las alternativas de tratamiento disponibles para su recuperación y de los riesgos que ello pueda representar, así como del pronóstico esperado... de acuerdo con su edad y condición personal y emocional" (Art. $8^{\circ}$ ).

La noción de consentimiento informado se encuentra actualmente en el centro de la medicina. El respeto hacia el paciente siempre ha presidido la práctica de la medicina, pero con la peculiaridad de que la capacidad de las personas para consentir o decidir no recibía un valor ético significativo. No nos estamos limitando a un acto aislado y puntual, como puede ser la aceptación de una prueba o un tratamiento. Nos estamos refiriendo a algo más amplio, a un modo de entender la relación clínica como un diálogo entre agentes morales: el reto que plantea el consentimiento informado es fundamentalmente ético. Entre la medicina paternalista y la medicina comercializada o defensiva, está una medicina de rostro humano con decisiones compartidas, que es la que deseamos promover desde la bioética.

\section{Competencia e información al menor en la Ley chilena}

En los anteriores proyectos de $\mathrm{Ley}^{8}$ se recogía que el médico debía actuar con el consentimiento del menor maduro, de 14 a 17 años, y no podría informar a los padres sin su consentimiento, salvo casos de intervenciones invasivas o de mayor riesgo.

Ha desaparecido en la Ley totalmente el consentimiento del menor. Lo único que dice es: “Toda persona tiene derecho a ser informada, en forma oportuna y comprensible, por parte del médico u otro profesional tratante (...), de acuerdo con su edad y condición personal y emocional." (Art. 10) Según esta redacción, se deja al arbitrio prudente del médico tratante la decisión de si conviene o no informar y a quienes. Parece muy débil la falta de argumentación, que no recoge el consenso general sobre la gradual competencia del menor maduro.

Las normas éticas pueden guiar al médico en el trato con menores, con el respeto a su autonomía que conlleva a la vez el deber de promover su competencia ${ }^{9}$. Por eso se utiliza generalmente la "regla ética del menor maduro": el análisis de la naturaleza de la determinación de capacidad, apoyada también por los estudios empíricos de las facultades de toma de decisiones de los niños y por la teoría del desarrollo, concluye que se presume su competencia y hay que justificar la incompetencia ${ }^{10: 251}$, e intervenir siempre en el mejor interés del menor ${ }^{11}$.

\section{El rechazo de tratamientos y las voluntades anticipadas}

La Ley recoge únicamente la posibilidad del rechazo en situación terminal "a cualquier tratamiento que tenga como efecto prolongar artificialmente su vida, sin perjuicio de mantener las medidas de soporte ordinario. En ningún caso, el rechazo de tratamiento podrá implicar como objetivo la aceleración artificial del proceso de muerte" (Art. 16º.

De todas formas, aparece claramente al hablar del consentimiento informado que el paciente puede rechazar un tratamiento -no sólo en situaciones terminales-, que no puede imponérsele por parte del médico (Art. 14\%), y que puede solicitar el alta voluntaria (Art. $16^{\circ}, \mathrm{p} 2^{\circ}$ ). Desde la ética, el paciente puede tener motivos muy válidos para rechazar un tratamiento propuesto, por situación económica, familiar, valores y creencias propios, etc $^{12}$. Debe verificarse su competencia si existen dudas, sobre todo si puede haber riesgo grave, $y$ debe informársele bien, pero sin coacciones, promoviendo su competencia y su decisión voluntaria, en lo que se considera una "muerte digna", alejada de toda posible eutanasia ${ }^{13}$.

En cuanto a las voluntades anticipadas, o instrucciones previas, la confusión entre algunas situaciones clínicas - rechazo de tratamientos, limitación de tratamientos, peticiones de eutanasialleva a algunos al rechazo sin más de las voluntades anticipadas, o impulsa a otros a defenderlas como "paso previo" a una posible legalización de la eutanasia. Ambas posiciones están erradas a nuestro parecer, pues pensamos que hay que entender la declaración anticipada de voluntades como un paso positivo más en el proceso de consenti- 
Ley derechos y deberes de las personas en atención de salud - F. J. León C.

miento informado del paciente ${ }^{14}$, especialmente en atención primaria ${ }^{15}$, sin convertirlas sólo en un documento más para la toma de decisiones médicas en situaciones críticas.

Un acierto de la Ley, a nuestro parecer, es modificar lo que estaba establecido sobre la certificación de muerte cerebral. Hasta ahora, solamente servía ese criterio en caso de donación de órganos para trasplante, pero no se decía nada en caso de no ser donante. Se establece (Art. 19 ${ }^{\circ}$ ) que el criterio de muerte encefálica, de todo el cerebro y el tronco cerebral, da la mejor certeza científica por el momento, y la suficiente certeza moral como para determinar que la persona ya ha fallecido y extender el certificado de defunción. Se están manteniendo artificialmente el funcionamiento de algunos órganos o sistemas, pero el paciente ya ha fallecido. Si alguien opina en conciencia en contrario y no tiene esa certeza moral, podrá realizar objeción de conciencia, pero la Ley salvaguarda la certeza moral de la gran mayoría de los médicos.

\section{Desarrollo de los Comités de Ética Asistencial (CEA)}

La Ley le concede más atribuciones a los CEA. Aunque sus dictámenes son de asesoría y no de obligatorio cumplimento, sí es obligatoria la consulta -hasta ahora solamente se sugería consultaren el caso de que los familiares quieran limitar el tratamiento y el médico piense que todavía es proporcionado (art. $17^{\circ}$ ), cuando la dirección de la institución quiera emitir un alta forzosa (art. $18^{\circ}$ ), o en casos de esterilizaciones, psicocirugías y otros tratamientos irreversibles a pacientes con discapacidad mental (art. 24\%), en que será necesario un informe favorable del CEA.

Tal y como se plantea en la Ley, será cada vez más necesaria la presencia de los CEA en todas las instituciones de salud. Los dilemas éticos de cada institución deben resolverse dentro de la misma, y además los CEA tienen el deber esencial de velar por el cumplimiento de los derechos de los pacientes.

\section{Investigación y personas con discapacidad psíquica o intelectual}

La ley establece una serie de medidas para proteger a las personas con discapacidad psíquica o intelectual, en casos de internamiento o trata- miento involuntario, medidas de aislamiento, etc. Y crea una Comisión Nacional de Protección de los Derechos de las Personas con Enfermedades Mentales (art. 29). El objetivo es muy loable, pero es bastante complicado su cumplimiento, si de verdad quieren seguir el tratamiento ambulatorio, hospitalización o participación en investigaciones, de estos pacientes en todo el país. Quizás puedan cumplimentar sus objetivos si se apoyan en los Comités de Ética Asistencial y los Comités de Ética Científicos de Investigación que ya existen, y que de hecho tienen la misma misión de velar por los derechos de los pacientes y sujetos de investigación, y especialmente de los más vulnerables.

Quisiéramos destacar el artículo $28^{\circ}$ de la Ley, que establece de forma taxativa:"ninguna persona con discapacidad psíquica o intelectual que no pueda expresar su voluntad podrá participar en una investigación científica". Es un exceso de protección que no tiene en cuenta las normas éticas internacionales que regulan la investigación con personas vulnerables ${ }^{16}$. Existe acuerdo -salvo algunas pocas excepciones- en que no se pueden investigar en ellos otras patologías, pero sí las suyas propias, como único modo de progresar en su tratamiento ${ }^{17}$.

\section{Más allá de los deberes de los pacientes}

Se exponen finalmente los deberes de los pacientes: respetar el reglamento interno del establecimiento de salud, informarse acerca de su funcionamiento y de los procedimientos de consulta y reclamo establecidos, cuidar las instalaciones y equipamiento, tratar respetuosamente al equipo de salud, y finalmente, colaborar informando de manera veraz acerca de sus necesidades y problemas de salud y de todos los antecedentes para su adecuado diagnóstico y tratamiento.

La Ley establece algunos deberes de los profesionales y de las instituciones para con los pacientes, pero no entra en los derechos y deberes de los profesionales frente a la institución, ni en los derechos y deberes de las instituciones frente al sistema de salud. Hay que definir bien los derechos de los médicos, la defensa de sus propias convicciones clínicas y éticas, como, por ejemplo, con la objeción de conciencia en algunos casos y las condiciones éticas de su trabajo en las instituciones de salud, que de hecho permitan una relación personalizada y humanizada. 


\section{Conclusiones}

En definitiva, bienvenida la aportación positiva de esta Ley, aun con todos los defectos que hemos señalado, que pensamos deben resolverse en los reglamentos, y sobre todo con la creación de la Comisión Nacional de Bioética que debería funcionar ya desde el año 2006 y debe asesorar al poder ejecutivo, legislativo y judicial en este ámbito. Pero no debemos olvidar que el modelo de relación médico-paciente que se propone desde la bioética, de decisiones compartidas, va más allá de unas relaciones de no maleficencia y justicia y de la aplicación estricta de unos derechos y deberes. Esta Ley comienza a atender, aunque tímidamente, a la autonomía de los pacientes, pero debemos señalar que nos queda mucho por delante en la humanización de la atención en salud, en la excelencia de los cuidados con los pacientes, en la calidad asistencial y en un profesionalismo médico que tenga en cuenta la competencia, pero también los valores de servicio, beneficencia y solidaridad social.

\section{Referencias}

1. Giedion U, Villar M, Ávila A. Los Sistemas de Salud en Latinoamérica y el papel del Seguro Privado. Madrid: Fundación Mapfre; 2010. Estudio sobre Chile: 87-104.

2. Erazo A. Universalidad efectiva y protección social. El desafío de las reformas sociales. En: Erazo X, Martín MP, Oyarze H (Ed.) Políticas públicas para un Estado social de derechos. Santiago: Lom Ediciones y Fundación Henry Dunant América Latina; 2007: 265-89.

3. Olabarría M (Editor) ¿Cómo se formulan las políticas públicas de salud en Chile? Santiago: Editorial Universitaria; 2012.

4. Proyecto de Ley de derechos y deberes de los pacientes. Mensaje presidencial No 038-344 para la presentación ante la Cámara de Diputados, 2001.

5. Erazo A, Titelman D. Cobertura y garantías en salud. En: Erazo X, Abramovich V, Orbe J (Edit.) Políticas públicas para un Estado social de derechos. El paradigma de los derechos universales. Santiago: LOM Ediciones, Henry Dunant América Latina; 2008: 253-71.

6. Bascuñán ML. Cambios en la relación médico-paciente y nivel de satisfacción de los médicos. Rev Med Chile 2005; 133: 11-6.

7. Kottow M. Consideraciones bioéticas sobre la Ley de derechos y deberes en atención de salud. Cuadernos Médico-Sociales 2012; 52 (1): 22-7.

8. Texto del Primer Informe Proyecto de Ley que regula los derechos y deberes que las personas tienen en relación con acciones vinculadas a su atención de salud. Boletín $\mathrm{n}^{\circ}$ 4.398-11. Artículos $8^{\circ}$ y $10^{\circ}$.

9. McNab ME, Beca JP. ¿Existen límites en la decisión de los padres sobre el tratamiento de sus hijos? Rev Chil Pediat 2010; 81 (6): 536-40.

10. Buchanan AE, Brock DW. Decidir por otros. Ética de la toma de decisiones subrogada. México: UNAM y Fondo de Cultura Económica; 2009.

11. Kottow M, Saada A. Participación informada en clínica e investigación biomédica. Las múltiples facetas de la decisión y el consentimiento informado. Bogotá: RedBioética UNESCO y Universidad Nacional de Colombia; 2007.

12. Beca JP, Ortiz A, Solar S. Derecho a morir: un debate actual. Rev Med Chile 2005; 133: 601-6.

13. Goic A. Apuntes sobre la eutanasia. Rev Med Chile 2005; 133: 371-5.

14. Siurana JC. Voluntades anticipadas. Madrid: Trotta; 2006.

15. Santos C. Documento de voluntades anticipadas: actitud de los pacientes de atención primaria. Rev Aten Primaria, 2003; 32 (1): 1-8.

16. Lolas F, Quezada A, Editores. Pautas éticas de investigación en sujetos humanos: nuevas perspectivas. Santiago: Programa Regional de Bioética OPS/OMS; 2003.

17. AMM. Declaración de Helsinki. 2008. Art. $27^{\circ}$ y $28^{\circ}$. 